\title{
04.1;04.4
}

\section{Пылевые звуковые солитоны в плазме запыленной экзосферы Луны}

\author{
() С.И. Копнин, С.И. Попель
}

Институт космических исследований РАН,

Москва, Россия

E-mail: serg_kopnin@mail.ru

Поступило в Редакцию 17 декабря 2020 г.

В окончательной редакции 2 февраля 2021 г.

Принято к публикации 6 февраля 2021 г.

Показана возможность существования и распространения пылевых звуковых солитонов в запыленной плазме экзосферы Луны, содержащей помимо электронов и ионов солнечного ветра и фотоэлектронов от ее поверхности заряженные пылевые частицы, а также фотоэлектроны, эмитирующие с поверхностей этих частиц. Найдены солитонные решения и определены диапазоны возможных скоростей и амплитуд таких солитонов в зависимости от высоты над лунной поверхностью для различных угловых высот Солнца.

Ключевые слова: пылевая плазма, пылевые звуковые солитоны, пылевая звуковая мода, адиабатически захваченные электроны.

DOI: 10.21883/PJTF.2021.09.50904.18660

В последние годы во всем мире усиливается интерес к исследованиям Луны. Особенно следует отметить успехи КНР в развитии лунных программ (см., например, [1]). Исследования процессов, протекающих на поверхности Луны, также крайне важны, особенно ввиду предстоящих российских лунных миссий, таких как ,Луна-25“, „Луна-26“, „Луна-27“ (см., например, [2]) и т.д. Особый интерес представляют процессы, связанные с наличием пылевых частиц в экзосфере Луны [3]. Лунная поверхность покрыта пылевыми частицами, возникшими в результате бомбардировки метеоритами различных размеров на протяжении многих миллиардов лет. Поверхность Луны, обращенная к Солнцу, находится под воздействием солнечного ветра и солнечной радиации. При этом лунная поверхность приобретает положительный заряд (важную роль играет фотоэффект) [4]. При определенных условиях силы электростатического отталкивания могут привести к подъему пылевых частиц субмикро- и микромасштабных размеров над лунной поверхностью [5]. В этом случае можно говорить о „запыленной“ экзосфере Луны [6], в которой помимо электронов и ионов солнечного ветра содержатся левитирующие заряженные пылевые частицы, а также фотоэлектроны, поступающие в результате фотоэмиссии с лунной поверхности и с поверхностей левитирующих пылевых частиц. Следует подчеркнуть, что роль фотоэлектронов в процессе зарядки пылевых частиц оказывается определяющей. На рис. 1 приведены параметры „запыленной“ экзосферы Луны, полученные на основе численных расчетов в модели, описанной в [5].

Настоящая работа посвящена определению возможности распространения пылевых звуковых солитонов и нахождению их параметров (характерных диапазонов скоростей и амплитуд) в условиях таким образом „запыленной“ экзосферы Луны.

К образованию пылевых звуковых солитонов у освещенной стороны Луны может, например, приводить пылевая звуковая неустойчивость, которая достаточно легко реализуется в условиях плазмы в области взаимодействия хвоста магнитосферы с Луной [7,8]. Если в результате раскачки колебаний (или волны) вследствие

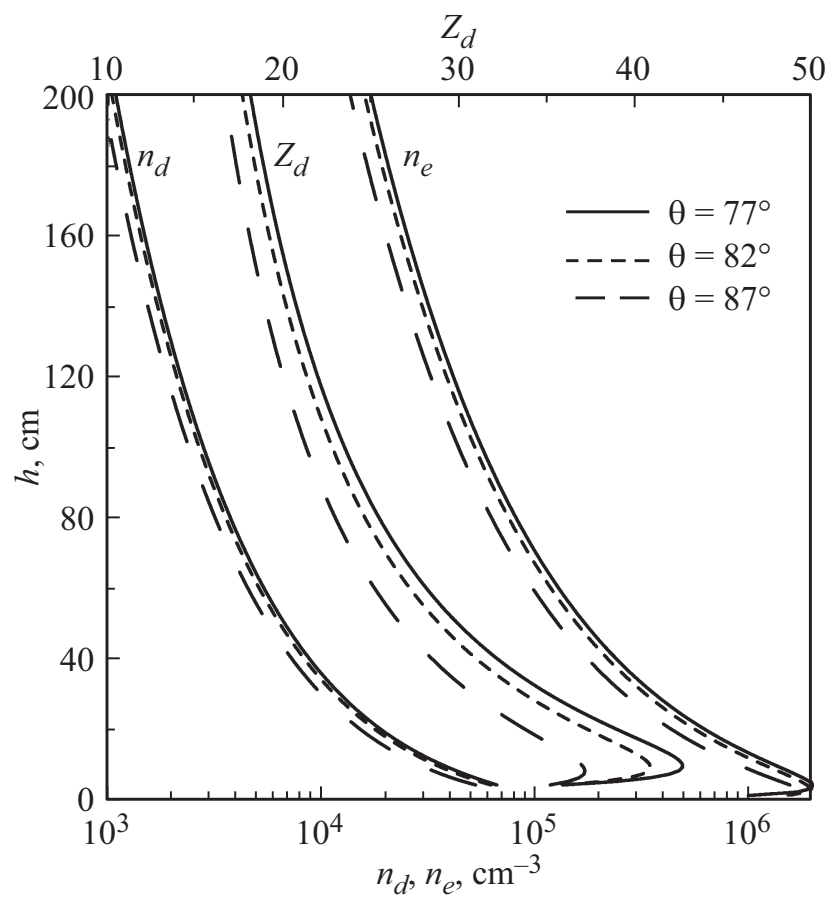

Рис. 1. Параметры „запыленной“ экзосферы Луны в зависимости от высоты $h$ над лунной поверхностью $\left(n_{e}\right.$ и $n_{d}-$ концентрации электронов и пылевых частиц, $Z_{d}-$ заряд пылевой частицы, выраженный в количестве электронов). 
развития неустойчивости их амплитуды становятся достаточно большими, такими, что уже невозможно линейное рассмотрение, то в плазме могут возникать пылевые звуковые нелинейные волны, одним из видов которых являются солитоны.

При описании пылевых звуковых солитонов (см., например, $[9,10])$ важную роль играет динамика пылевых частиц, которую можно описать с помощью уравнения непрерывности и уравнения движения (Эйлера)

$$
\begin{gathered}
\partial n_{d} / \partial t+\partial\left(n_{d} v_{d}\right) / \partial x=0 \\
\partial v_{d} / \partial t+v_{d}\left(\partial v_{d} / \partial x\right)=-\left(q_{d} / m_{d}\right)(\partial \varphi / \partial x),
\end{gathered}
$$

где $\varphi-$ самосогласованный потенциал в плазме, координата $x$ имеет горизонтальное направление, $n_{d}-$ концентрация пылевых частиц, $-e-$ заряд электрона, $q_{d}=Z_{d}$ е и $m_{d}-$ заряд $\left(Z_{d}-\right.$ заряд пылевой частицы, выраженный в количестве электронов) и масса пылевой частицы, $v_{d}-$ ее направленная скорость. Уравнения (1) справедливы, когда распространение солитонов горизонтально и, кроме того, высоты $h$, на которых рассматривается распространение солитонов, значительно превосходят дебаевский радиус электронов плазмы $\lambda_{D e}=\sqrt{T_{e} / 4 \pi n_{e} e^{2}}$. Здесь $n_{e}-$ концентрация электронов, $T_{e}$ - температура электронов, выраженная в энергетических единицах.

На временны́х масштабах, характерных для пылевых звуковых колебаний, инертностью электронов и ионов можно пренебречь. Поэтому электроны и ионы успевают подчиниться статистическим распределениям. В таких условиях (когда пылевые частицы приобретают положительный заряд в результате действия фотоэффекта) пылевые звуковые солитоны создают положительный электростатический потенциал [11], который представляет собой потенциальную яму для электронов. Электроны могут быть захвачены такой потенциальной ямой, когда выполнено следующее неравенство [12]:

$$
\tau_{S} \geq l_{S} / v_{T e},
$$

где $\tau_{S}, l_{S}$ - характерные пространственно-временны́е масштабы солитона, $v_{T e}$ - тепловая скорость электронов. Отметим, что $\tau_{S} \propto \omega_{p d}^{-1}$ (где $\omega_{p d}=\sqrt{4 \pi n_{d} q_{d}^{2} / m_{d}}-$ пылевая плазменная частота), пространственный размер солитона $l_{S}$ составляет величину порядка нескольких дебаевских радиусов электронов. Следовательно, $l_{S} / v_{T e} \propto \omega_{p e}^{-1}$ (где $\omega_{p e}=\sqrt{4 \pi n_{e} e^{2} / m_{e}}-$ плазменная частота электронов, здесь $m_{e}-$ масса электрона). Для пылевых звуковых солитонов неравенство (2) справедливо практически всегда. Поэтому при описании электронов следует учитывать их адиабатический захват [12] потенциальной ямой, сформированной пылевым звуковым солитоном, и в этом случае распределение электронов описывается формулой Гуревича

$$
n_{e}=n_{e 0}\left[\left(1-\frac{2}{\sqrt{\pi}} \int_{0}^{\sqrt{e \varphi / T_{e}}} e^{-u^{2}} d u\right) e^{\frac{e \varphi}{T_{e}}}+\frac{2}{\sqrt{\pi}} \sqrt{\frac{e \varphi}{T_{e}}}\right]_{(3)}
$$

Поскольку потенциал в пылевом звуковом солитоне положительный, для ионов подобного захвата не происходит, и можно считать, что ионы подчинены распределению Больцмана

$$
n_{i}=n_{i 0} e^{-\frac{e \varphi}{T_{i}}}
$$

Здесь $n_{i}$ - концентрация ионов, $T_{i}$ - температура ионов, выраженная в энергетических единицах, индекс 0 соответствует невозмущенным состояниям.

Систему уравнений (1), (3) и (4) следует дополнить уравнением Пуассона

$$
\partial^{2} \varphi / \partial x^{2}=4 \pi e\left(n_{e}-n_{i}-n_{d} Z_{d}\right) .
$$

Систему уравнений (1), (3)-(5) можно решать методом сагдеевского потенциала. Для локализованного волнового решения, распространяющегося с некоторой постоянной скоростью $M$, можно перейти в новую систему координат $\xi=x-M t$. В этом случае все параметры задачи будут зависеть только от новой переменной $\xi$. Сагдеевский потенциал в безразмерных переменных
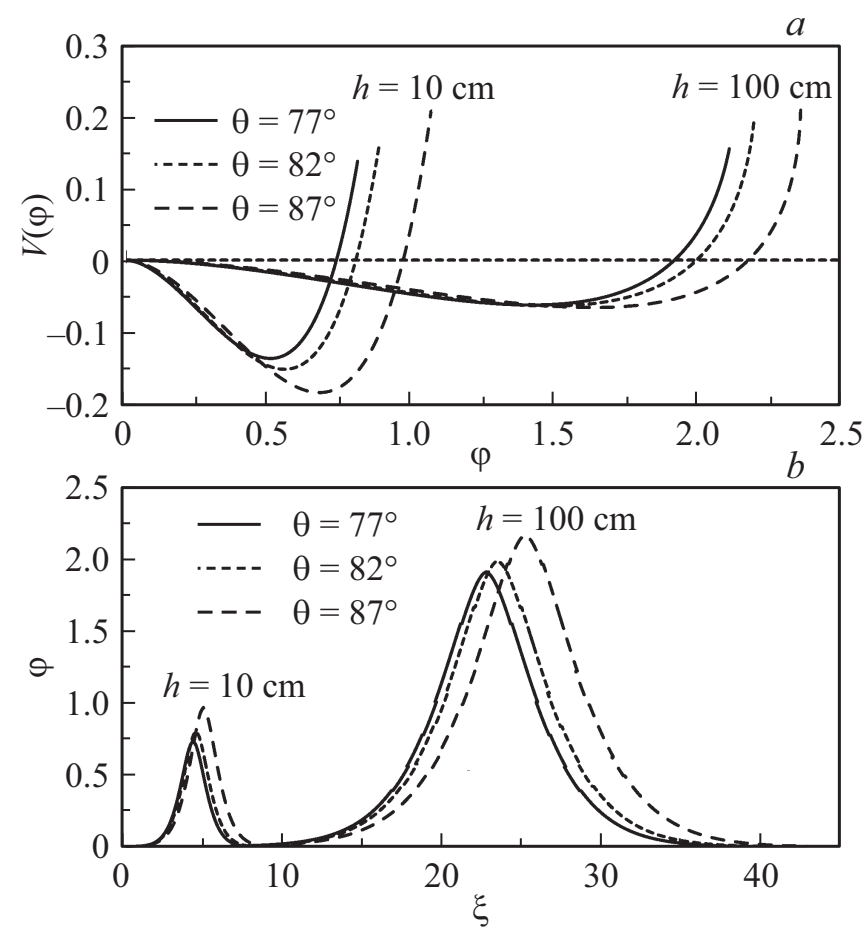

Рис. 2. Характерные пылевые звуковые солитоны и сагдеевские потенциалы для параметров плазмы, соответствующих высотам $h=10$ и $100 \mathrm{~cm}$ и скорости $M=10$ для всех профилей. 


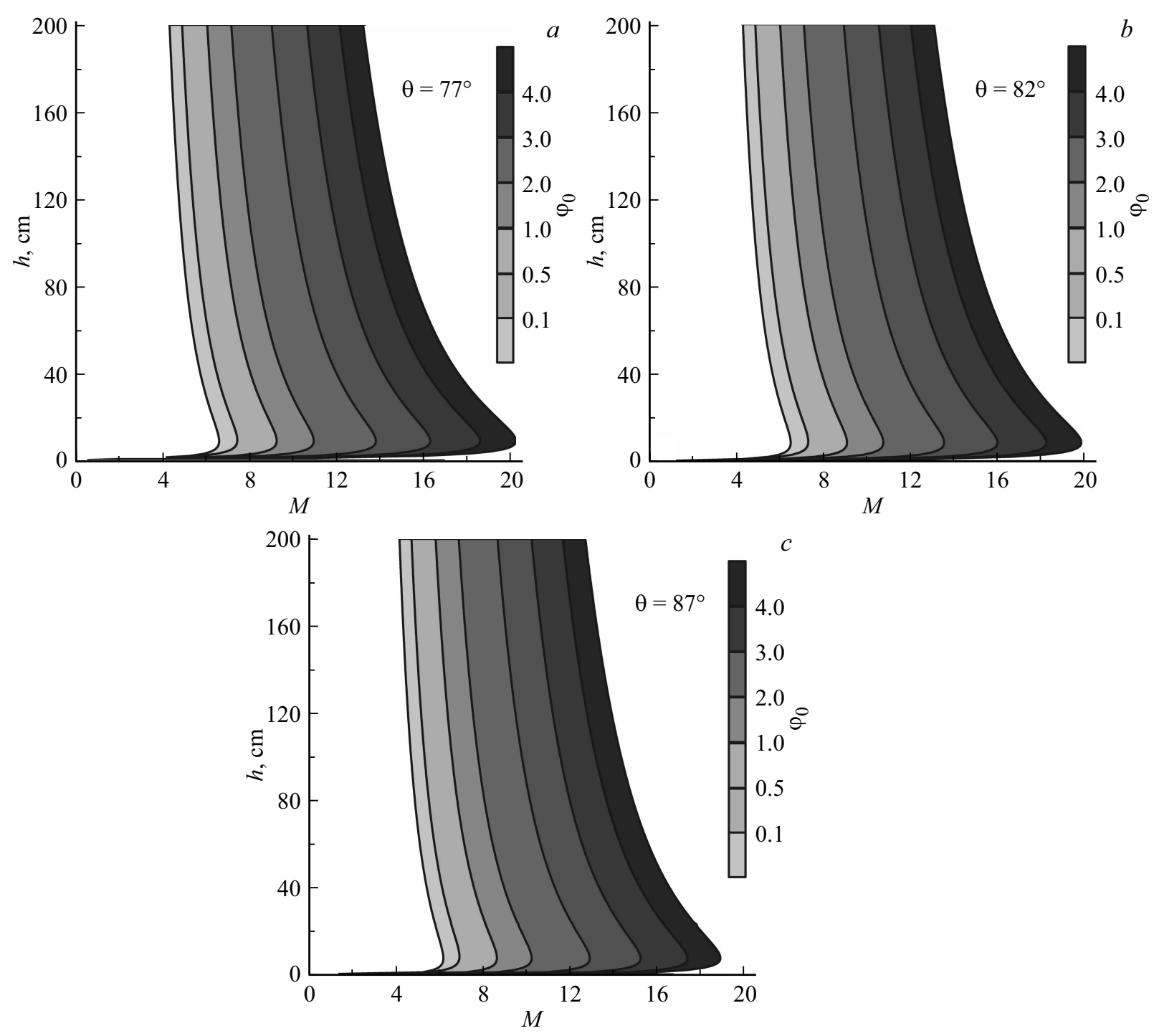

Рис. 3. Профили амплитуд для пылевых звуковых солитонов в зависимости от высоты $h$ над поверхностью Луны для всего возможного диапазона скоростей $M$, когда угловая высота Солнца $\theta=77^{\circ}(a), 82^{\circ}(b)$ и $87^{\circ}(c)$.

$e \varphi / T_{e} \rightarrow \varphi, M / C_{S d} \rightarrow M, \xi / \lambda_{D e} \rightarrow \xi$, где $C_{S d}=\sqrt{T_{e} / m_{d}}$, принимает следующий вид (ср., например, с [13] и [14]):

$$
\begin{aligned}
V(\varphi)= & {\left[t(h, \theta)+Z_{d}(h) d(h)\right]\left[1-e^{\varphi}\left(1-\frac{2}{\sqrt{\pi}}\right.\right.} \\
& \left.\left.\times \int_{0}^{\sqrt{\varphi}} \exp \left(-u^{2}\right) d u\right)-2 \sqrt{\varphi / \pi}-4 \sqrt{\varphi^{3} / 9 \pi}\right] \\
& +M d(h)\left(M-\sqrt{M^{2}-2 Z_{d} \varphi}\right),
\end{aligned}
$$

где $t(h, \theta)=\cos \theta\left[1+h \lambda_{D e}^{-1} \sqrt{\cos \theta / 2}\right]^{-2}-$ слагаемое, соответствующее фотоэлектронам от лунной поверхности, $d(h)=n_{d}(h) / n_{e 0}$, концентрация фотоэлектронов у поверхности Луны [15] $n_{e 0}=2.1 \cdot 10^{5} \mathrm{~cm}^{-3}, \theta-$ угловая высота Солнца над лунной поверхностью. Считаем, что температура всех фотоэлектронов составляет величину около $0.15 \mathrm{eV}$. Диапазон скоростей $\left(M_{\min }<M<M_{\max }\right)$ для пылевых звуковых солитонов ограничивается следующими выражениями:

$$
\begin{aligned}
& M \geq M_{\min }=\sqrt{Z_{d}^{2}(h) d(h) /\left[t(h, \theta)+Z_{d}(h) d(h)\right]} \\
& {\left[t(h, \theta) / d(h)+Z_{d}(h)\right]\left[1-\exp \left(M_{\max }^{2} / 2 Z_{d}(h)\right)\left(1-\frac{2}{\sqrt{\pi}}\right.\right.} \\
& \left.\times \int_{0}^{\sqrt{M_{\max }^{2} / 2 Z_{d}(h)}} \exp \left(-u^{2}\right) d u\right)-2 \sqrt{M_{\max }^{2} / 2 \pi Z_{d}(h)} \\
& \left.-4 \sqrt{\left[M_{\max }^{2} / 2 Z_{d}(h)\right]^{3} / 9 \pi}\right]+M^{2} \geq 0 .
\end{aligned}
$$

На рис. 2 представлены характерные солитоны и сагдеевские потенциалы для параметров плазмы, соответствующих высотам $h=10$ и $100 \mathrm{~cm}$ (см. рис. 1) и скорости 
$M=10$. На рис. 3 приведены профили амплитуд для пылевых звуковых солитонов в зависимости от высоты $h$ над поверхностью Луны для всего возможного диапазона скоростей $M$, когда угловая высота Солнца $\theta=77^{\circ}(a), 82^{\circ}(b)$ и $87^{\circ}(c)$.

Отметим, что для всего диапазона параметров плазмы запыленной экзосферы Луны амплитуды пылевых звуковых солитонов оказываются того же знака, что и величина электрического заряда пылевых частиц, т.е. при $Z_{d} e>0$ амплитуда солитона $\varphi_{0}>0$.

Таким образом, в запыленной экзосфере Луны, содержащей электроны и ионы солнечного ветра, заряженные пылевые частицы и фотоэлектроны, эмитирующие с лунной поверхности и поверхностей пылевых частиц, могут существовать локализованные волновые структуры, связанные с пылевой звуковой модой, такие как пылевые звуковые солитоны. Оказывается, что в этом случае следует учитывать адиабатический захват электронов потенциальными ямами, образованными такими солитонами. Методом сагдеевского потенциала найдены солитонные решения и определены диапазоны возможных скоростей и амплитуд таких солитонов в зависимости от высоты над лунной поверхностью для различных угловых высот Солнца. Выше параметры солитонов приведены в безразмерном виде. Реальные амплитуды солитонов, которые могли бы быть зарегистрированы в будущих наблюдениях, соответствуют значениям электрического поля порядка $1-10 \mathrm{~V} / \mathrm{m}$. При этом характерная ширина солитона составляет величину порядка $1 \mathrm{~cm}-1 \mathrm{~m}$.

\section{Конфликт интересов}

Авторы заявляют, что у них нет конфликта интересов.

\section{Список литературы}

[1] D. Li, Y. Wang, H. Zhang, X. Wang, Y. Wang, Z. Sun, J. Zhuang, C. Li, L. Chen, H. Zhang, X. Zou, C. Zong, H. Lin, J. Ma, X. Li, X. Cui, R. Yao, X. Wang, X. Gao, Sh. Yang, X. Wang, B. Zhang, Geophys. Res. Lett., 47 (17), e2020GL089433 (2020) https://doi.org/10.1029/2020GL089433

[2] Л.М. Зеленый, С.И. Попель, А.В. Захаров, Физика плазмы, 46 (5), 441 (2020). DOI: 10.31857/S0367292120050108 [Пер. версия: 10.1134/S1063780X20050104].

[3] А.В. Захаров, Л.М. Зеленый, С.И. Попель, Астрон. вестн., 54 (6), 483 (2020). DOI: 10.31857/S0320930X20060079

[4] E. Walbridge, J. Geophys. Res., 78(19), 3668 (1973). https://doi.org/10.1029/JA078i019p03668

[5] С.И. Попель, С.И. Копнин, А.П. Голубь, Г.Г. Дольников, А.В. Захаров, Л.М. Зеленый, Ю.Н. Извекова, Астрон. вестн., 47 (6), 455 (2013). https://doi.org/10.7868/S0320930X13060078

[6] А.П. Голубь, Г.Г. Дольников, А.В. Захаров, Л.М. Зеленый, Ю.Н. Извекова, С.И. Копнин, С.И. Попель, Письма в ЖЭТФ, $95(4), 198$ (2012). [Пер. версия: $10.1134 / \mathrm{S} 0021364012040054]$.
[7] С.И. Попель, Т.И. Морозова, Физика плазмы, 43 (5), 474 (2017). DOI: 10.7868/S0367292117050079 [Пер. версия: $10.1134 /$ S1063780X17050075].

[8] Yu.N. Izvekova, T.I. Morozova, S.I. Popel, IEEE Trans. Plasma Sci., 46 (4), 731 (2018). https://doi.org/10.1109/TPS.2017.2752084

[9] S.I. Kopnin, I.N. Kosarev, S.I. Popel, M.Y. Yu, Planet. Space Sci., 52 (13), 1187 (2004). https://doi.org/10.1016/j.pss.2004.09.003

[10] S.I. Popel, S.I. Kopnin, I.N. Kosarev, M.Y. Yu, Adv. Space Res., 37 (2), 414 (2006). https://doi.org/10.1016/j.asr.2005.12.003

[11] Т.И. Морозова, С.И. Копнин, С.И. Попель, Физика плазмы, 41 (10), 867 (2015). DOI: 10.7868/S0367292115100066 [Пер. версия: 10.1134/S1063780X15100062].

[12] Л.Д. Лившиц, Л.П. Питаевский, Теоретическая физика. T. 10. Физическая кинетика (Физматлит, М., 2002), c. 182.

[13] С.И. Копнин, И.Н. Косарев, С.И. Попель, Ю. Минг, Физика плазмы, 31 (3), 224 (2005). [Пер. версия: https://doi.org/10.1134/1.1884685]

[14] С.И. Копнин, С.И. Попель, Письма в ЖТФ, $45(20)$, 26 (2019). DOI: 10.21883/PJTF.2019.20.48389.17837 [Пер. версия: $10.1134 / \mathrm{S} 1063785019100237]$.

[15] Е.К. Колесников, А.С. Мануйлов, Астрон. журн., 59(5), 996 (1982). 